\title{
Surface Modification of Aluminum Plate with Fluoroalkyl End-capped Acrylic Acid Oligomer/silica Nanocomposites - Oleophobic to Hydrophilic Switching Behavior Adapted to the Environmental Change on the Modified Plate Surface
}

\author{
By Hiroshi KAKEHI, ${ }^{1,2}$ Masashi MUIRA, ${ }^{2}$ Norifumi ISU, ${ }^{2}$ and Hideo SAWADA ${ }^{1, *}$
}

Fluoroalkyl end-capped acrylic acid oligomer/silica nanocomposites were prepared by reaction of the corresponding oligomer with silica nanoparticles in the presence of alkaline silicate in water. Fluorinated oligomer/silica nanocomposites thus obtained are nanometer size-controlled fine particles $(48-67 \mathrm{~nm})$ and were found to exhibit a good dispersibility in their aqueous solutions, although the silica composites, which were prepared in the absence of oligomer, afforded submicrometer size-controlled particles $(197 \mathrm{~nm}$ ). These fluoroalkyl end-capped oligomer/silica nanocomposites were applied to the surface modification of aluminum plate. Interestingly, the modified plate surface exhibits a strong oleophobicity imparted by fluorine. On the other hand, this modified plate surface was found to exhibit a good hydrophilicity related to the presence of carboxyl groups in composites when the environment is changed from air to water on the surface.

KEY WORDS: Fluorinated Oligomer / Hydrophilicity / Oleophobicity / Surface Modification / Aluminum Plate / Silica Nanocomposite /

SEM / DLS / Contact Angle /

There has been considerable interest in polymeric surfactants (polysoaps) which exhibit a variety of unique properties such as high dispersing, aggregates and emulsion properties that set them apart from the corresponding low-molecular weight surfactants. ${ }^{1}$ In general, these polysoaps have a poor surface active characteristic compared to the low-molecular weight surfactants. ${ }^{2}$ From the developmental viewpoint of polysoaps possessing a surface active properties, it is of considerable interest to prepare fluoroalkylated polysoaps. In fact, there have been numerous studies on the synthesis and surfactant properties of randomly fluoroalkylated and AB block-type fluoroalkylated polysoaps. ${ }^{3}$ In these fluorinated polysoaps, AB block-type fluorinated polysoaps can exhibit a higher surface active characteristic than that of randomly ones, and these fluorinated polyasoaps were applied to the surface modification to exhibit a surface active property imparted by fluorine on the surface. ${ }^{4}$ In our comprehensive studies on the synthesis and properties of novel fluoroalkylated polysoaps, we have already demonstrated that partially fluoroalkylated, in particular, ABA triblock-type fluoroalkyl end-capped oligomers are attractive materials, because they exhibit various unique properties such as high solubility, surface active properties, and nanometer sizecontrolled self-assembled molecular aggregates which cannot be achieved by the corresponding non-fluorinated, randomly and $\mathrm{AB}$ block-type fluoroalkylated polymers. ${ }^{5}$ In these fluoroalkyl end-capped oligomers, fluoroalky end-capped oligomers containing hydrophilic segments such as morpholino and carboxyl groups are applicable to the surface modification of traditional organic polymers such as poly(methyl methacrylate) to have a good hydrophilicity with a good oil repellency. ${ }^{6}$ However, the adhesion ability between the PMMA film surface and fluorinated oligomer is extremely poor. Therefore, the exploration of fluoroalkyl end-capped oligomers possessing not only a high surface-active characteristic but also good adhesion ability toward the parent plate surface is deeply desirable from the practical points of view. In fact, we have recently succeeded in preparing fluoroalkyl end-capped oligomers/silica nanocomposites possessing a good adhesion ability toward the glass plate. ${ }^{7}$ It is strongly suggested that fluoroalkyl end-capped oligomer/silica nanocomposites containing hydrophilic segments should exhibit not hydro- and oleo-phobic characteristics imparted by fluoroalkyl segments in composites but hydrophobic to hydrophilic switching behavior on the surface when the surface environment is changed from air to water. Thus, these fluorinated nancomposites would have high potential for the practical applications into a wide variety of fields as a new fluorinated surface modifier. In fact, it was previously reported that polyurethanes containing perfluoropolyether, polydimethylsiloxane and polyethylene glycol segments, and poly(styrene$g$-ethylene oxide) having fluorinated polyethylene oxide chain ends can exhibit oleophobic, hydrophobic, and hydrophilic properties in response to the polarity of the contacting medium. ${ }^{8,9}$ In this paper, we would like to demonstrate on the surface modification of aluminum plate with fluoroalkyl endcapped acrylic acid oligomer/silica nanocomposites, with particular emphasis on the hydrophobic to hydrophilic switching behavior adapted to environmental change on the modified plate surface.

\footnotetext{
${ }^{1}$ Department of Frontier Materials Chemistry, Graduate School of Science and Technology, Hirosaki University, Hirosaki 036-8561, Japan ${ }^{2}$ General Research Institute of Technology, INAX Corporation, Tokoname, Aichi 479-8588, Japan

*To whom correspondence should be addressed (Tel: +81-172-39-3578, Fax: +81-172-39-3578, E-mail: hideosaw @ cc.hirosaki-u.ac.jp).
} 


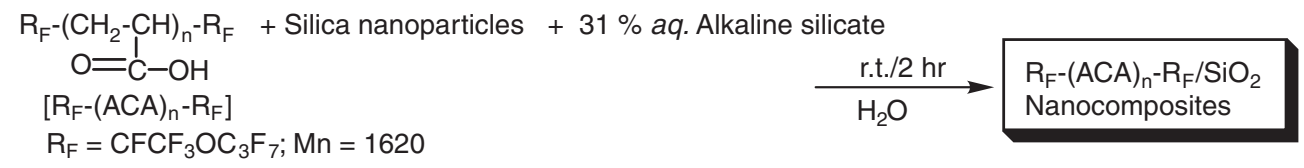

Scheme 1.

\section{EXPERIMENTAL}

\section{Measurements}

Molecular weight of oligomer was measured using a Shodex DS-4 (pomp) and Shodex RI-71 (detector) gel permeation chromatography (GPC) calibrated with polystyrene standard using tetrahydrofuran as the eluent. Dynamic light-scattering (DLS) were measured by using Otsuka Electronics DLS-7000 HL (Tokyo, Japan). Field Emission-Scanning electron microscopy (FE-SEM) images were measured by using a HITACHI S-4700 (Tokyo, Japan). Contact angles were measured by the use of Kyowa Interface Science CA-X and DM-500 (Saitama, Japan). AFM (atomic force microscope) were recorded on a SII NanoTechnology SPA-300HV (Tokyo, Japan).

\section{Materials}

Fluoroalkyl end-capped acrylic acid oligomer $\left[\mathrm{R}_{\mathrm{F}}-(\mathrm{ACA})_{\mathrm{n}^{-}}\right.$ $\mathrm{R}_{\mathrm{F}}$ ] was prepared by reaction of fluoroalkanoyl peroxide with acrylic acid monomer according to our previously reported method. ${ }^{10}$ Silica nanoparticles (average particle size: $12 \mathrm{~nm}$ ) was purchased from Nippon Aerosil Co., Ltd. (Tokyo, Japan). Alkaline silicate and silica particles were purchased from Nippon Chemical Industrial Co., Ltd. (Tokyo, Japan) and Marukamakamado Touryou Co., Ltd. (Gifu, Japan), respectively. Methyltrimethoxysilane, trimethylmethoxysilane and (heptadecafluoro-1,1,2,2-tetrahydrodecyl)trimethoxysilane were purchased from Shin-Etsu Chemical Co., Ltd. (Tokyo, Japan). The aluminum plate $(100 \mathrm{~mm} \times 100 \mathrm{~mm} \times 1 \mathrm{~mm})$ used was commercial grade: JIS A1100.

\section{Preparation of Fluoroalkyl End-capped Acrylic Acid Oligomer/silica Nanocomposites}

Following is a typical experimental procedure for the preparation of fluorinated silica nanocomposites. To an aqueous solution (45 $\mathrm{g}$ ) of fluoroalkyl end-capped acrylic acid oligomer $\left[\mathrm{R}_{\mathrm{F}}-\left(\mathrm{CH}_{2} \mathrm{CHCOOH}\right)_{n}-\mathrm{R}_{\mathrm{F}} \quad\left[\mathrm{R}_{\mathrm{F}}-(\mathrm{ACA})_{\mathrm{n}}-\mathrm{R}_{\mathrm{F}}\right] ; \mathrm{R}_{\mathrm{F}}=\right.$ $\mathrm{CF}\left(\mathrm{CF}_{3}\right) \mathrm{OC}_{3} \mathrm{~F}_{7} ; \mathrm{Mn}=1620$ (24 mg) were added silica nanoparticles (average particle size: $12 \mathrm{~nm} ; 2.0 \mathrm{~g}$ ) and $31 \%$ aqueous alkaline silicate ( $32 \mathrm{~g})$. The mixture was stirred with a magnetic stirring bar at room temperature for $2 \mathrm{~h}$ to afford the transparent composite solution. The dynamic light scattering (DLS) measurements at $25^{\circ} \mathrm{C}$ show that the size $(67 \mathrm{~nm})$ of the obtained composites is nanometer size-controlled very fine particles.

\section{Surface Modification of Aluminum Plate with $\mathbf{R}_{\mathrm{F}}-(\mathbf{A C A})_{\mathbf{n}^{-}}$ $\mathbf{R}_{\mathbf{F}}$ /silica Nanocomposites}

Firstly, the pre-modified aluminum plate was prepared by the spray coating of a well-dispersed aqueous solution (130 g)
Table I. Preparation of $R_{F-}(A C A)_{n}-R_{F} /$ silica nanocomposites

\begin{tabular}{ccccc}
\hline Run & $\begin{array}{c}\mathrm{R}_{\mathrm{F}}-(\mathrm{ACA})_{\mathrm{n}}-\mathrm{R}_{\mathrm{F}} \\
(\mathrm{mg})\end{array}$ & $\begin{array}{c}\text { Silica } \\
\text { nanoparticles } \\
(\mathrm{g})\end{array}$ & $\begin{array}{c}31 \% \text { aq. Alkaline } \\
\text { silicate } \\
(\mathrm{g})\end{array}$ & $\begin{array}{c}\text { Particles Size of } \\
\left.\text { the composite }{ }^{\mathrm{b}}\right) \\
(\mathrm{nm})\end{array}$ \\
\hline 1 & 24 & 2 & 32 & $66.7 \pm 8.9$ \\
2 & 60 & 2 & 32 & $57.8 \pm 10.6$ \\
3 & 120 & 2 & 32 & $47.9 \pm 4.3$ \\
\hline
\end{tabular}

a) Determined by dynamic light scattering measurements.

containing alkaline silicate ( $16 \mathrm{~g})$ and silica particles (average particle size: $3 \mu \mathrm{m} ; 40 \mathrm{~g})$. The above mentioned $R_{F}-(A C A)_{n}-$ $\mathrm{R}_{\mathrm{F}}$ /silica nanocomposite solution was applied to the surface modification of this pre-modified aluminum plate by the use of the similar spray coating technique. The modified aluminum plate thus obtained was dried at $180^{\circ} \mathrm{C}$ for $2 \mathrm{~h}$, and the contact angles for oleic triglyceride and water of the surface of this plate (plate size: $10 \mathrm{~mm} \times 10 \mathrm{~mm}$ ) were measured at room temperature by the use of the contact angle measurements.

\section{RESULTS AND DISCUSSION}

Fluoroalkyl end-capped acrylic acid oligomer reacted with silica nanoparticles in the presence of alkaline silicates in aqueous solutions to afford the nanometer size-controlled composites under very mild conditions. These results were shown in Scheme 1 and Table I.

As shown in Scheme 1 and Table I, the size (numberaverage diameter) of the obtained fluorinated oligomer/silica composites by DLS measurements at $25^{\circ} \mathrm{C}$ was found to decrease (67 to $48 \mathrm{~nm}$ ) effectively with the increase of the used amounts of $R_{\mathrm{F}}-(\mathrm{ACA})_{\mathrm{n}}-\mathrm{R}_{\mathrm{F}}$ oligomer from 24 to $120 \mathrm{mg}$. On the other hand, the size of the composites was found to increase significantly to $197 \mathrm{~nm}$ in the absence of the corresponding oligomer. The increase size $(48-67 \mathrm{~nm})$ of fluorinated oligomer/silica nanocomposites, compared to that $(12 \mathrm{~nm})$ of the used parent silica nanoparticles indicates that the composization of silica nanoparticles with fluoroalkyl end-capped oligomer could proceed smoothly to afford very fine fluorinated oligomer/silica nanocomposite particles.

We have measured SEM (scanning electron microscopy) photographs of $\mathrm{R}_{\mathrm{F}}-(\mathrm{ACA})_{\mathrm{n}}-\mathrm{R}_{\mathrm{F}} /$ silica nanocomposites in water, and the results were shown in Figures 1-3. SEM images also showed the formation of fluorinated silica composite (Figures 1-3) nanoparticles with a mean diameter of $31 \mathrm{~nm}$ (Figure 1), $20 \mathrm{~nm}$ (Figure 2) and $16 \mathrm{~nm}$ (Figure 3), and we could obtain the similar average particle size in SEM and DLS measurements, respectively. DLS and SEM measurements show that the self-assembled oligomeric aggregates formed by 


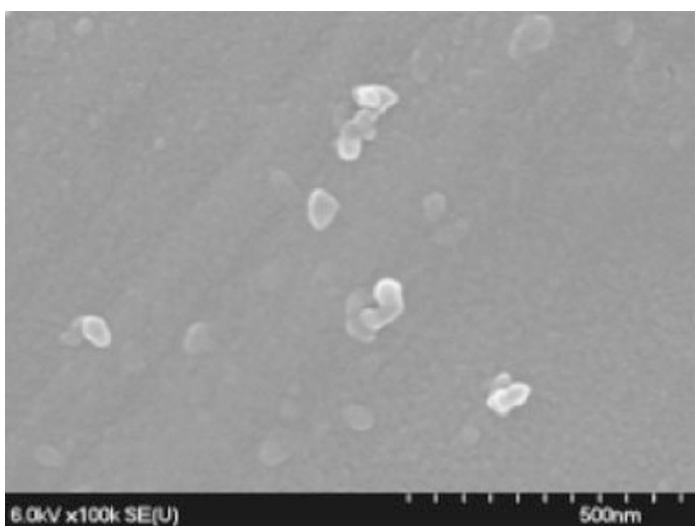

Figure 1. SEM (scanning electron microscopy) images of $R_{F}-(A C A)_{n}-R_{F} /$ $\mathrm{SiO}_{2}$ nanocomposites in water (Run 1 in Table I).

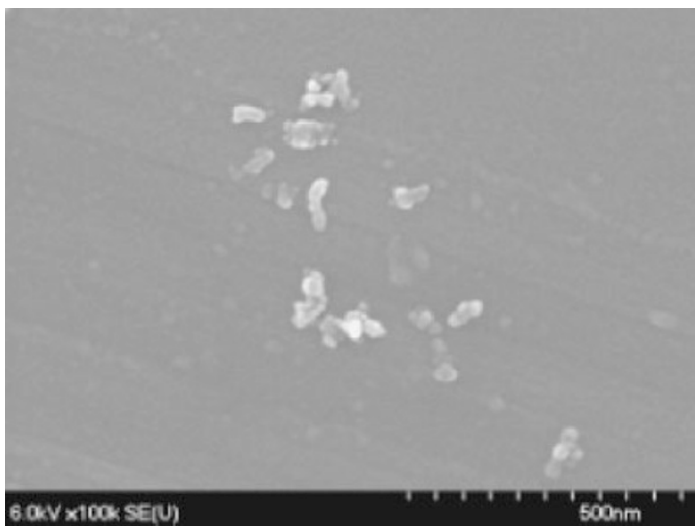

Figure 2. SEM (scanning electron microscopy) images of $R_{F}-(A C A)_{n}-R_{F} /$ $\mathrm{SiO}_{2}$ nanocomposites in water (Run 2 in Table I).

$\mathrm{R}_{\mathrm{F}}-(\mathrm{ACA})_{\mathrm{n}}-\mathrm{R}_{\mathrm{F}}$ oligomer in alkaline silicate solutions should interact smoothly with silica nanoparticles to afford very fine nanocomposite particles.

Our present fluorinated oligomer/silica nanocomposites in Table I was found to afford transparent solutions. Thus, it is in particular interest to develop our fluorinated silica nanocomposites to the surface modification for the aluminum plates. We have prepared the modified aluminum plates treated with fluorinated oligomers/silica nanocomposites listed in Table I, and the contact angles of oleic triglyceride and water on the modified aluminum plates were measured, and these results were shown in Figure 4.

The contact angle of oleic triglyceride on the aluminum plate surface treated with fluorinated oligomer/silica nanocomposites (contents of oligomer based on the used alkaline silicates and silica nanoparticles are $0.2,0.5$ and $1.0 \%$, corresponding to Run 1, Run 2 and Run 3 in Table I, respectively) showed a significantly large value $\left(62-63^{\circ}\right)$ under atmospheric conditions in each case, indicating that the modified aluminum surfaces could exhibit a good oleophobicity imparted by fluoroalkyl segments in nanocomposites (see Figure 4a). In contrast, the contact angle of oleic triglyceride on the aluminum surface treated with silica composites

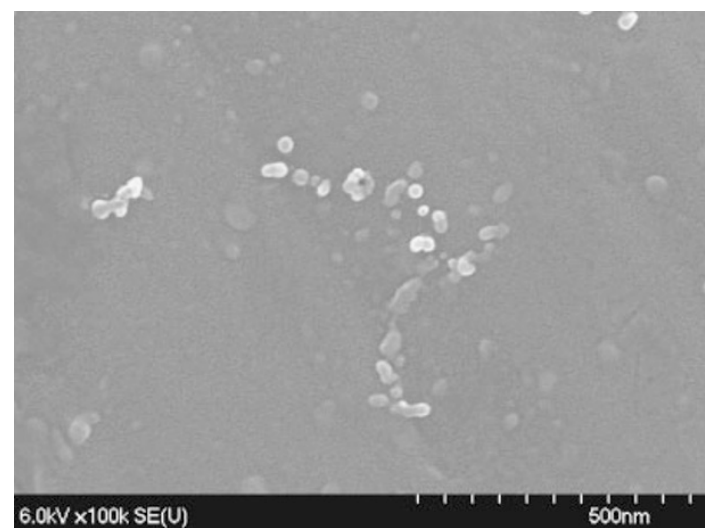

Figure 3. SEM (scanning electron microscopy) images of $R_{F}-(A C A)_{n}-R_{F} /$ $\mathrm{SiO}_{2}$ nanocomposites in water (Run 3 in Table I).

(a)

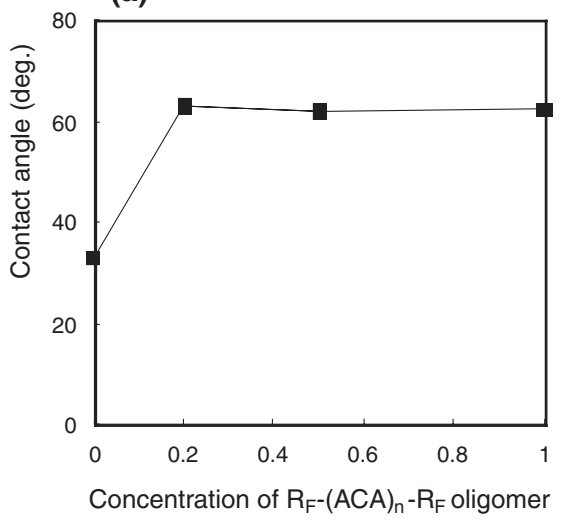

(b)

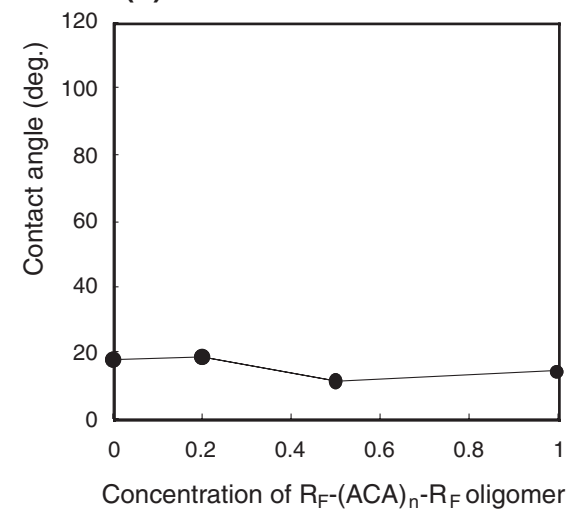

Figure 4. Relationship between the contact angle values of oleic triglyceride (a) or water (b) and the concentration of $R_{F}-(A C A)_{n}-R_{F}$ oligmer in silica nanocomposites.

containing no oligomer exhibited a smaller value $\left(32^{\circ}\right)$ to afford an oleophilic characteristic.

Contact angle value of water on modified aluminum plates with fluorinated nanocomposites was from 12 to $19^{\circ}$ in each concentration of oligomer in nanocomposites, indicating that these modified surface can exhibit a strong hydrophilic characteristic (see Figure 4b). The contact angle value $\left(18^{\circ}\right)$ of water on the modified aluminum plate treated with silica 
(a)

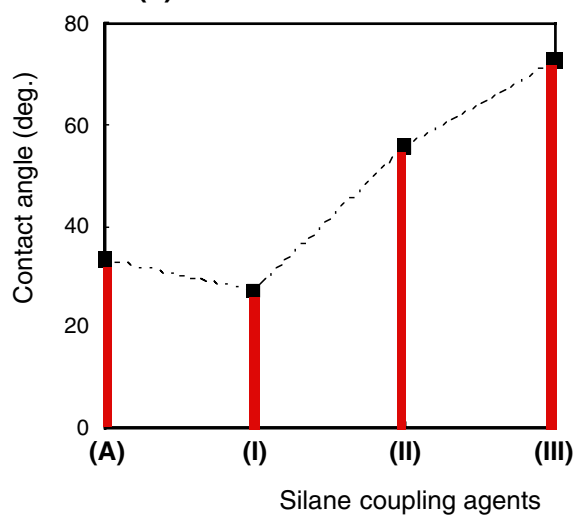

(b)

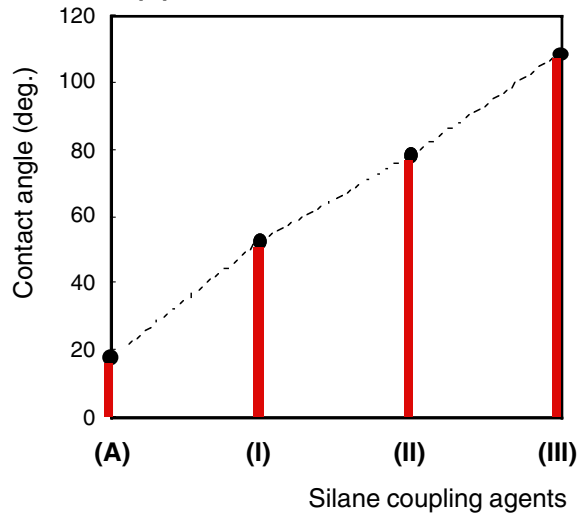

Figure 5. Contact angle values of oleic triglyceride (a) and water (b) on the modified aluminum plates treated with methyltrimethoxysilane (I)/, trimethylmethoxysilane (II)/, and F-Si (III)/silica nanocomposites:

(A) indicates the modified aluminum plate with silica composites containing no silane coupling agent.

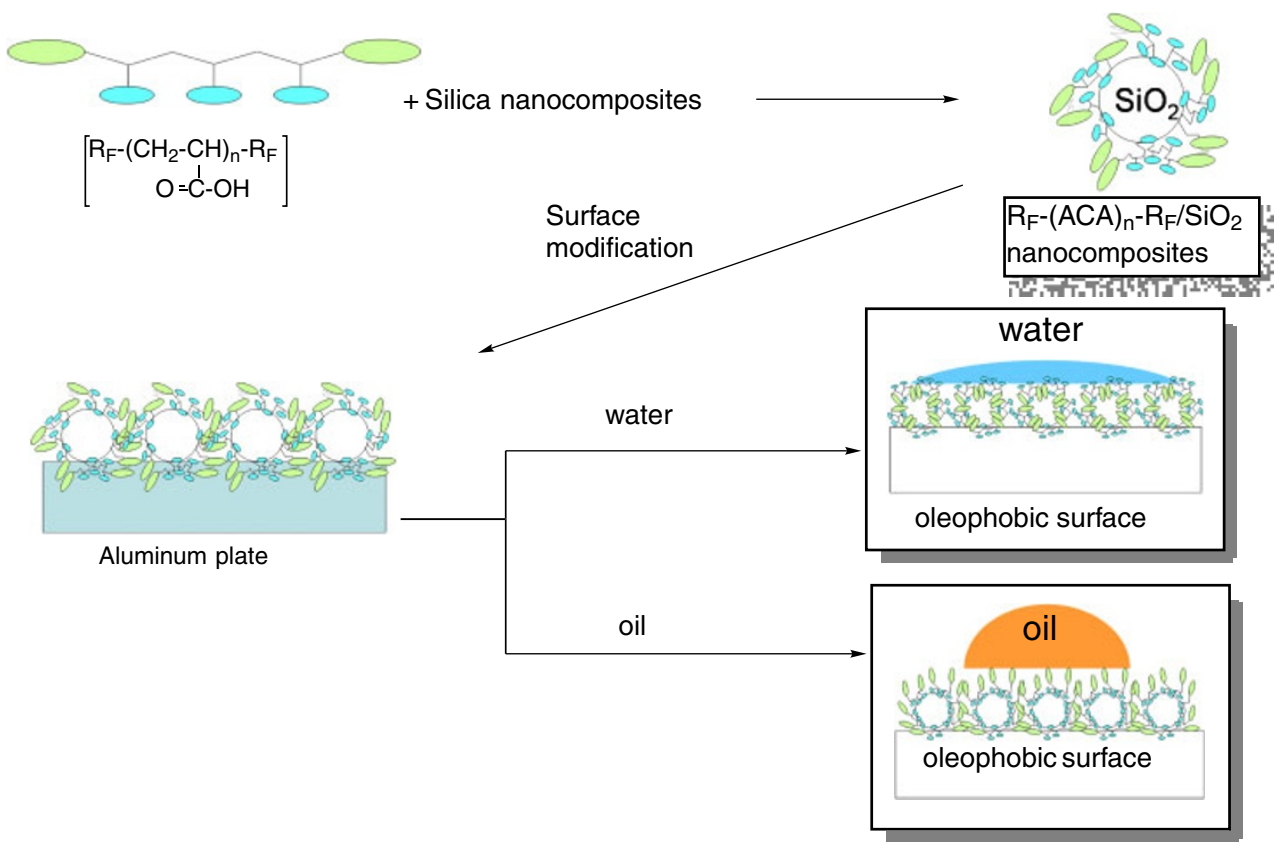

Scheme 2.

composite containing no oligomer also exhibit a hydrophilic characteristic due to the presence of hydrophilic silanol segments in silica composites.

We have measured the contact angle values of oleic triglyceride and water under atmospheric conditions on the modified aluminum plates treated with methyltrimethoxysilane/silica composite, trimethylmethoxysilane/silica composite and (heptadecafluoro-1,1,2,2-tetrahydrodecyl)trimethoxysilane $(\boldsymbol{F}$-Si $) /$ silica composite, which were prepared under similar conditions as that of Table I, and the results were shown in Figure 5.

As shown in Figure 5a, we have a higher contact angle value $\left(73^{\circ}\right)$ of oleic triglyceride in the case of $\boldsymbol{F}$-Si $/$ silica composite. Similarly, we could observe a higher contact angle value of water $\left(109^{\circ}\right)$ in the case of $\boldsymbol{F}-\mathbf{S i}$, compared to those $\left(50-80^{\circ}\right)$ of other traditional silane coupling agents (see Figure 5b). Higher contact angle values of oleic triglyceride and water in the case of $\mathbf{F - S i} /$ silica composite are due to the presence of longer fluoroalkyl groups in $\boldsymbol{F}$-Si, which can exhibit strong oleophobic and hydrophobic characteristics. On the other hand, as shown in Figure $4 a$, our present $\mathrm{R}_{\mathrm{F}}-(\mathrm{ACA})_{\mathrm{n}}-\mathrm{R}_{\mathrm{F}}$ / silica nanocomposites could afford a strong oleophobic characteristic related to the presence of fluoroalkyl groups in composites on the modified surface under the atmospheric conditions. However, interestingly, when the environment is changed from air to the water on the modified surface, hydrophobic fluoroalkyl groups in silica nanocomposites should be easily replaced by the strongly hydrophilic carboxyl groups in oligomers at the interface with water to exhibit lower contact angle values of water around 12$19^{\circ}$ as illustrated in Scheme 2. In fact, as shown in Figure 6a, 
(b)

(a)
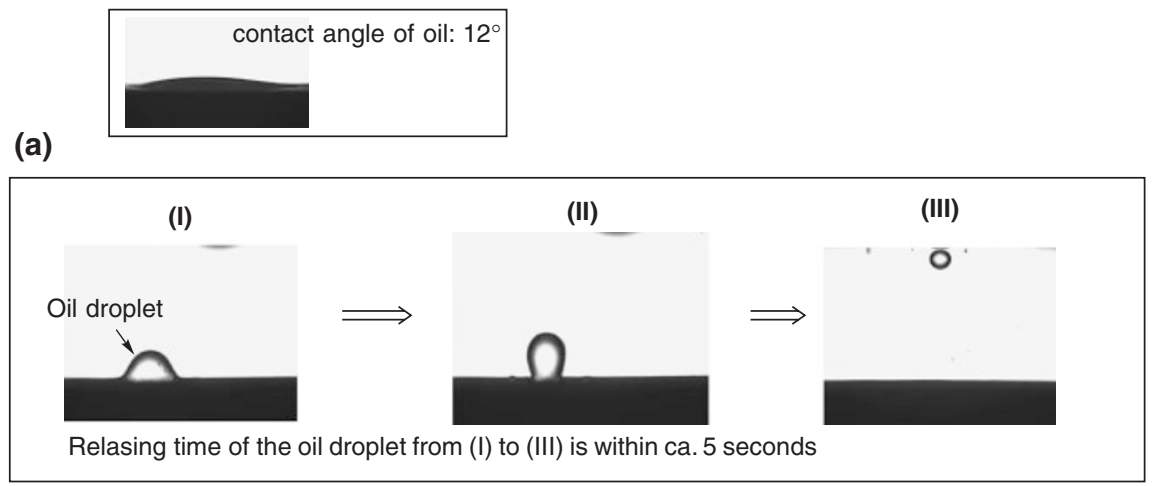

Figure 6. Photographys of releasing behaviors of oil droplets on the modified aluminum plate surfaces treated with fluorinated oligomer/silica nanocomposites (Run 3 in Table I) [(a)] and oil droplet on the modified aluminum plate treated with $\mathbf{F}$-Si/silica composites [(b)] after soaking these plates into the water bath:

Releasing time (from (I) to (III) of oil droplet from the surface is within around $5 \mathrm{~s}$.

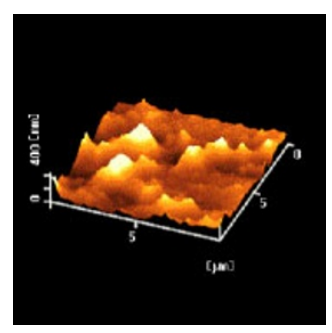

(a)

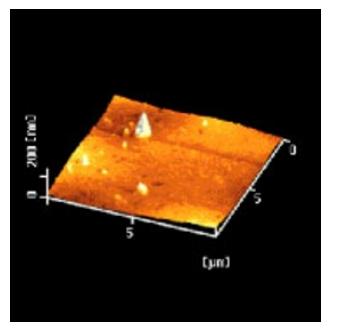

(b)

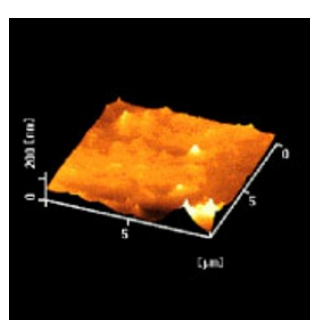

(c)

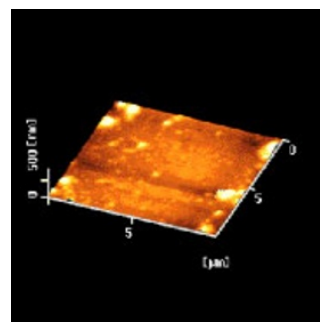

(d)

Figure 7. AFM topographic images of the modified aluminum plates surface treated with $R_{F}-(A C A)_{n}-R_{F} /$ silica nanocomposites
(a) parent aluminum plate
(b) modified aluminum plates surface treated with $R_{F}-(A C A)_{n}-R_{F} /$ silica nanocomposites (Run 1 in Table I)
(c) modified aluminum plates surface treated with $R_{F}-(A C A)_{n}-R_{F} /$ silica nanocomposites (Run 2 in Table I)
(d) modified aluminum plates surface treated with $R_{F}-(A C A) n-R_{F} /$ silica nanocomposites (Run 3 in Table I)

fluorinated silica nanocomposites could enable the oil droplet (oleic triglyceride) to release effectively from the modified aluminum plate surface by soaking this plate into the water bath, and this releasing time from (I) to (III) is within around $5 \mathrm{~s}$; although the corresponding $\boldsymbol{F}$-Si silica composite failed to release the oil droplet from the plate surface even after a few hours due to exhibiting a strong oleophilic characteristic (contact angle of oleic triglyceride: $12^{\circ}$ ) under similar conditions (see Figure 6b).

In general, the surface roughness is very important for the wetting properties for liquids. Thus, we have studied the surface roughness of these modified aluminum plates by the use of AFM (atomic force microscopy). AFM measurements showed that the roughness average: $\mathrm{Ra}(\mathrm{nm})$ of the parent aluminum plate surface and the modified aluminum plated surface treated with $\mathrm{R}_{\mathrm{F}}-(\mathrm{ACA})_{\mathrm{n}}-\mathrm{R}_{\mathrm{F}} /$ silica nanocomposites are as follows:

$\begin{array}{lc} & \mathrm{Ra}(\mathrm{nm}) \\ \text { Parent aluminum surface } & 45.5 \\ \text { Modified aluminum plates surface: } & \\ \text { Run 1 in Table I } & 9.38 \\ \text { Run 2 } & 14.2 \\ \text { Run 3 } & 10.5\end{array}$

Parent aluminum surface treated with alkaline silicate and silica naoparticles exhibits a higher $\mathrm{Ra}$ value, and the topographical images of this surface show a rough characteristic (see Figure 7a). However, unexpectedly, the modified aluminum plates treated with $\mathrm{R}_{\mathrm{F}}-(\mathrm{ACA})_{\mathrm{n}}-\mathrm{R}_{\mathrm{F}}$ / silica nanocomposites have extremely lower Ra values compared to that of the parent aluminum plate, indicating that these surface should show a smooth characteristic. In fact, a similar result was obtained in the topographic images of these surface in Figure $7 \mathrm{~b}, 7 \mathrm{c}$, and $7 \mathrm{~d}$. This finding suggests that the residual silanol groups on the aluminum plate surface, which was pre-modified with alkaline silicate and silica nanopaticle, should interact effectively with $\mathrm{R}_{\mathrm{F}}$-(ACA)- $\mathrm{R}_{\mathrm{F}}$ / silica nanocomposites to afford the corresponding modified aluminum plate surface possessing the smooth surface characteristic. Therefore, this smooth surface could afford a suitable oleophobic to hydrophilic switching behavior on the modified aluminum plate surface.

In this way, it was clarified that our present fluorinated nanocomposites are effectively bound to the aluminum plate surface. This suggests that silica nanoparticle units and some carboxyl groups in our fluoroalkyl end-capped acrylic acid oligomer/silica nanocomposites are very essential to the increase of the adhesion ability through the interaction with 


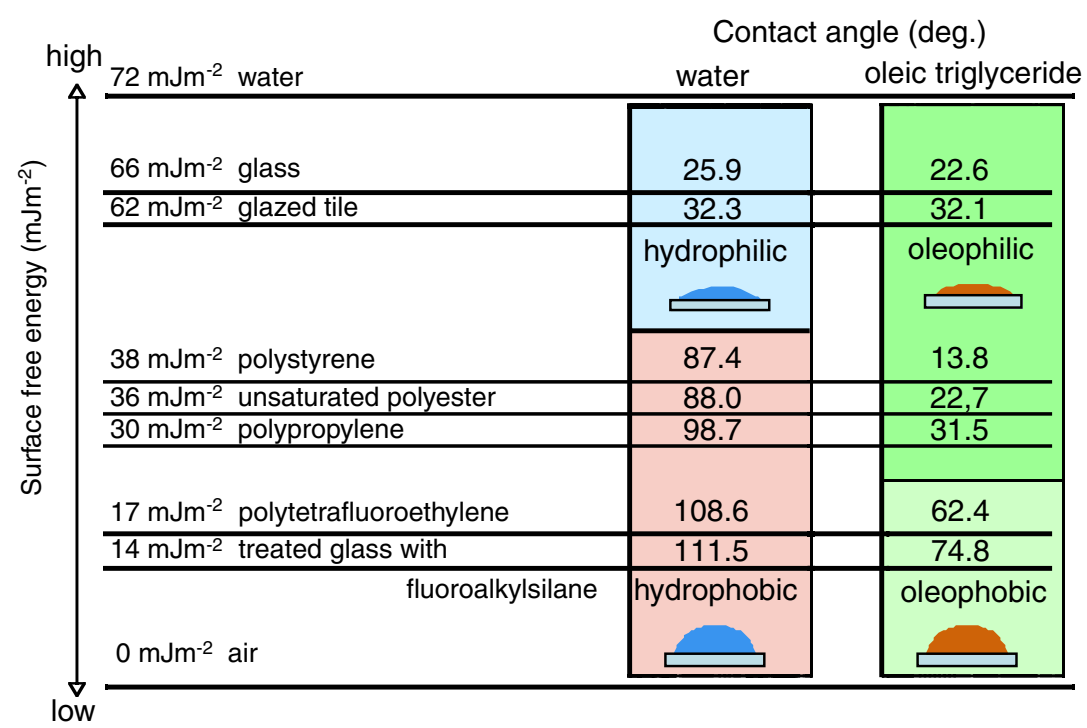

Figure 8. Surface free energy diagram of ceramics, organic polymer (polystyrene), and fluorinated material [poly(tetrafluoroethylene)], and contact angle values of water and oleic triglyceride on these material surface.

the residual silanol groups on the aluminum plate surface. Therefore, we can easily observe the oleophobic (by fluoroalkyl groups in nanocomposites) to hydrophilic (carboxyl groups in nanocomposites) switching behavior on the modified surface when the surface environment is changed from air to water, and it takes only a few seconds to replace the fluoroalkyl groups by carboxyl groups when the surface is in contact with liquid water. It is well-known that traditionally inorganic and organic materials such as ceramics, polystyrene, and poly(tetrafluoroethylene) can exhibit hydrophilic-oleophilic, hydrophobic-oleophilic, and hydrophobic-oleophobic characteristics, respectively, as illustrated in Figure 8. From the viewpoint of this surface free energy diagram, we have in general some difficulties to develop the materials possessing both hydrophilic and oleophobic characteristics. However, our present fluoroalkyl end-capped acrylic acid oligomer/silica nanocomposites have not only a strong oleophobic segments (fluoroalkyl groups) but also a strong hydrophilic segments (carboxyl groups) in oligomer main and side chains. This molecular design in our present nanocomposites could enable the modified plate surface to afford the novel hydrophobic to hydrophilic switching behavior. In particular, our present fluorinated silica nanocomposites have a remarkably strong adhesion ability toward the parent plate surface. Therefore, our present fluorinated silica nanocomposites have high potential for the applications to the surface modification in a wide variety of fields.

Acknowledgment. Thanks are due to Prof. Nagahiro Saitoh and Prof. Osamu Takai of Nagoya University for measurements of AFM.
Received: May 26, 2008

Accepted: August 1, 2008

Published: September 18, 2008

\section{REFERENCES}

1. B. Ameduri and B. Boutevin, "Well-Architectured Fluoropolymers: Synthesis, Properties and Applications," Elsevier, Amsterdam, 2004, pp231-348.

2. a) Y. Tanizaki, J. Jpn. Oil Chem. Soc., 34, 973 (1985).

b) P. Anton, O. Koberle, and A. Laschewsky, Makromol. Chem., 194, 1 (1993).

c) D. Cochin, P. Hendlinger, and A. Laschewsky, Colloid Polym. Sci., 273, 1138 (1995).

3. a) J.-F. Berret, D. Calvet, A. Collet, and M. Viguier, Curr. Opin. Colloid Interface Sci., 8, 296 (2003).

b) T. Imae, Curr. Opin. Colloid Interface Sci., 8, 307 (2003).

4. a) T. Imae, H. Tabuchi, K. Funayama, A. Sato, T. Nakamura, and N. Amaya, Colloid Surf. A: Physicochem. Eng. Aspects, 167, 73 (2000). b) M. Morita, M. Kubo, and M. Matsumoto, Colloid Surf. A, 109, 183 (1996).

c) I. J. Park, S.-B. Lee, C. K. Choi, and K.-J. Kim, J. Colloid Interface Sci., 181, 284 (1996).

d) I. J. Park, S.-B. Lee, and C. K. Choi, J. Appl. Polym. Sci., 54, 1449 (1994).

5. a) H. Sawada, Chem. Rev., 96, 1779 (1996).

b) H. Sawada, J. Fluorine Chem., 105, 219 (2000).

c) H. Sawada, Polym. J., 39, 637 (2007).

d) H. Sawada, Prog. Polym. Sci., 32, 509 (2007).

6. H. Sawada, K. Yanagida, Y. Inaba, M. Sugiua, T. Kawase, and T. Tomita, Eur. Polym. J., 37, 1433 (2001).

7. H. Sawada, T. Narumi, A. Kajiwara, K. Ueno, and K. Hamazaki, Colloid Polym. Sci., 284, 551 (2006).

8. A. Vaidya and M. K. Chaudhury, J. Colloid Interface Sci., 249, 235 (2002).

9. P. Jannasch, Macromolecules, 31, 1341 (1998).

10. H. Sawada, Y.-F. Gong, Y. Minoshima, T. Matsumoto, M. Nakayama, M. Kosugi, and T. Migita, J. Chem. Soc., Chem. Commun., 537 (1992). 